\title{
Imaging the two-component nature of Dirac-Landau levels in the topological surface state of $\mathrm{Bi}_{2} \mathrm{Se}_{3}$
}

\author{
Ying-Shuang Fu ${ }^{1 \dagger}$, M. Kawamura ${ }^{1}$, K. Igarashi ${ }^{2}$, H. Takagi ${ }^{3,4,5}$, T. Hanaguri ${ }^{1 \star}$ and T. Sasagawa ${ }^{2}$
}

\begin{abstract}
Massless Dirac electrons in condensed matter ${ }^{1-6}$ are, unlike conventional electrons, described by two-component wavefunctions associated with the spin degrees of freedom in the surface state of topological insulators ${ }^{5,6}$. Hence, the ability to observe the two-component wavefunction is useful for exploring novel spin phenomena. Here we show that the two-component nature is manifest in Landau levels, the degeneracy of which is lifted by a Coulomb potential. Using spectroscopic-imaging scanning tunnelling microscopy, we visualize energy and spatial structures of Landau levels in $\mathrm{Bi}_{2} \mathrm{Se}_{3}$, a prototypical topological insulator. The observed Landau-level splitting and internal structures of Landau orbits are distinct from those in a conventional electron system ${ }^{7}$ and are well reproduced by a two-component model Dirac Hamiltonian. Our model further predicts energy-dependent spin-magnetization textures in a potential variation and provides a way for manipulating spins in the topological surface state.
\end{abstract}

Landau quantization associated with the quasi-classical cyclotron motion of electrons in a magnetic field $B$ is a fundamental phenomenon and highlights the difference between conventional and Dirac electrons. In conventional systems, the energy of the $n$th Landau level $\left(\mathrm{LL}_{n}\right), E_{n}$, is proportional to $(n+\gamma) B$, where $\gamma=1 / 2$. Distinct from this, $E_{n}$ in two-dimensional massless Dirac systems behave as $\propto \sqrt{|n| B}$ (refs 8,9). Importantly, the Berry-phase effect in Dirac systems eliminates $\gamma$ and ensures the $B$-independence of $E_{0}$, which is equal to the Dirac-point energy ${ }^{10,11}$. Such an unusual LL sequence has been observed by scanning tunnelling microscopy and spectroscopy (STM/STS) in graphene $e^{12,13}$ and in the topological surface state ${ }^{14,15}$

In addition to the unique energy spectrum, the wavefunctions of Dirac LLs are remarkably different from their conventional counterparts because of their two-component nature ${ }^{9}$. To study the details of wavefunctions, spectroscopic-imaging STM (SI-STM) is a powerful technique because tunnelling-conductance maps, which include information of the internal structures of wavefunctions through local-density-of-states (LDOS) variations, can be obtained with high energy resolution and high spatial resolution. If the system is uniform, the spatial degeneracy of Landau orbits results in a homogeneous LDOS. The introduction of a potential variation lifts the spatial degeneracy, making it possible to access the localized Landau orbit ${ }^{16-19}$. The Landau orbit drifts along the equipotential lines and the LDOS variation across the orbit contains information of the internal structure of the wavefunction. Indeed, a recent SISTM study on a conventional two-dimensional electron system revealed the $n$-dependent nodal structure in the wavefunction ${ }^{7}$.

Wavefunction imaging could be even more interesting in Dirac systems, because a potential variation not only lifts the spatial degeneracy of Landau orbits but may also affect the interplay between the two components in the wavefunction. This is particularly important for the topological surface state, where the interplay determines the magnetic properties. Thus, exploring the two-component nature by wavefunction imaging will give us a clue towards developing a novel spin-manipulation protocol. For this, we study LL wavefunctions of a prototypical topological insulator $\mathrm{Bi}_{2} \mathrm{Se}_{3}$ using SI-STM.

Figure 1a represents LL spectra at $B=11 \mathrm{~T}$ taken at the marked points in the topographic image shown in Fig. 1b. We confirm that $E_{n}$ exhibits a dependence on $B$ and $n$ typical for Dirac electrons ${ }^{14,15}$. Moreover, the energy-momentum dispersion obtained by the scaling analysis ${ }^{14}$ agrees quantitatively with that obtained by angle-resolved photoemission spectroscopy on crystals from the same source ${ }^{20}$. These results guarantee that the obtained data faithfully represent intrinsic properties of the topological surface state (Supplementary Section 1.1). The potential landscape can be visualized by mapping the spatial variation of $E_{0}$. As $\mathrm{LL}_{0}$ is independent of $B$ and is located at the Dirac-point energy, the $E_{0}$ map faithfully represents the potential landscape, albeit it is smeared over the size of the $\mathrm{LL}_{0}$ wavefunction given by the magnetic length $l_{B}=\sqrt{\hbar /(|e| B)}$. Here, $\hbar$ is the Planck constant divided by $2 \pi$ and $e$ is the elementary charge. At $11 \mathrm{~T}, l_{B}$ is $\sim 7.7 \mathrm{~nm}$. As shown in Fig. 1c, there is a well-defined potential minimum in the field of view which may be generated by subsurface charged defects (such as Se vacancies). There is a line-shaped protrusion in the lower right corner of Fig. 1b, but it hardly affects the potential map shown in Fig. 1c. Potential variations with a similar length scale were also observed in graphene ${ }^{21}$ and doped topological insulators ${ }^{22}$.

We find that the potential-gradient map (Fig. 1d) exhibits strong correlation with the map of the apparent width of the $\mathrm{LL}_{0}$ peak (Fig. 1e), implying that the spatial variation of potential lifts the degeneracy of the LLs. This is clearly manifested in the individual tunnelling-conductance spectra shown in Fig. 1a. The $\mathrm{LL}_{0}$ peaks are sharp and single peaks at the potential minimum (blue) and at the edge of the potential dip where the potential becomes almost flat (red). At the potential-gradient maximum (green), the $\mathrm{LL}_{0}$ peak is not simply broadened but splits into multiple peaks which

${ }^{1}$ RIKEN Center for Emergent Matter Science, Wako, Saitama 351-0198, Japan, ${ }^{2}$ Materials and Structures Laboratory, Tokyo Institute of Technology, Yokohama, Kanagawa 226-8503, Japan, ${ }^{3}$ Magnetic Materials Laboratory, RIKEN, Wako, Saitama 351-0198, Japan, ${ }^{4}$ Department of Physics, University of Tokyo, Hongo, Bunkyo-ku, Tokyo 113-0033, Japan, ${ }^{5}$ Max-Planck-Institut für Festkörperforschung, Heisenbergstraße 1, 70569 Stuttgart, Germany. †Present address: School of Physics, Huazhong University of Science and Technology, Wuhan 430074, China. *e-mail: hanaguri@riken.jp 


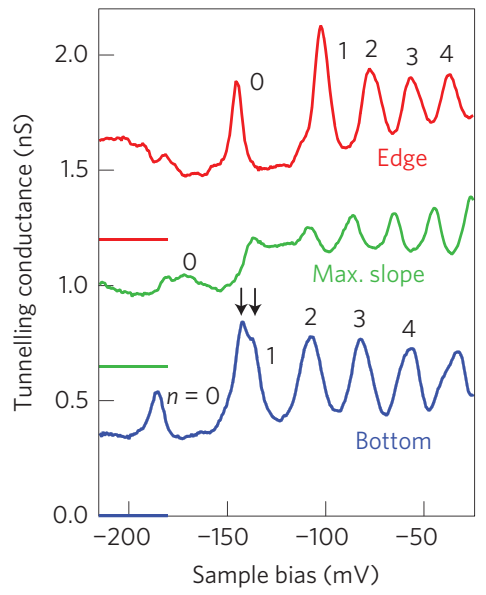

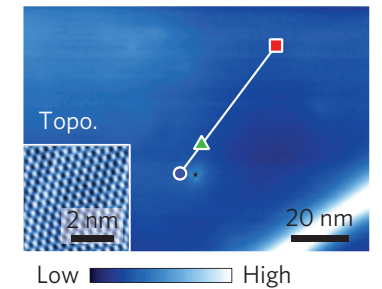

C

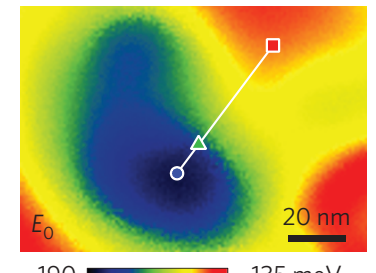

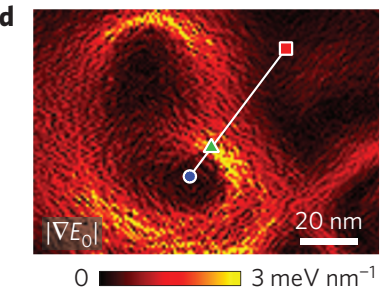

$0 \longleftarrow 3 \mathrm{meV} \mathrm{nm}^{-1}$

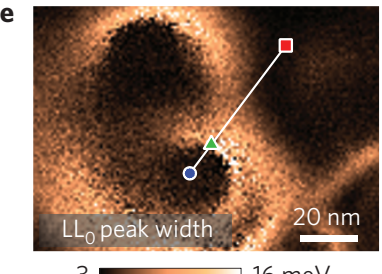

Figure 1 | Landau-level spectra at $11 \mathrm{~T}$ and potential landscape in the topological surface state of $\mathrm{Bi}_{2} \mathrm{Se}_{3}$. $\mathbf{a}$, Tunnelling spectra taken at representative

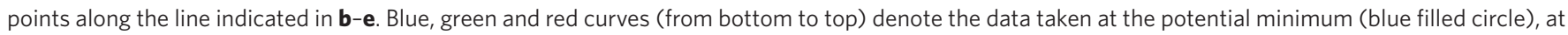

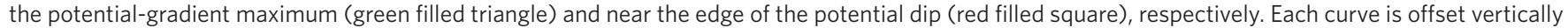
for clarity. The zero-line for each curve is denoted by a short horizontal line. Data were taken at $1.5 \mathrm{~K}$ with conditions of sample-bias voltage $V_{\mathrm{s}}=+50 \mathrm{mV}$, tunnelling current $I_{t}=50 \mathrm{pA}$ and bias modulation amplitude $V_{\text {mod }}=2.1 \mathrm{mV}$ rms. Note that the apparent LLo peak consists of a few peaks at the potential-gradient maximum and the $\mathrm{LL}_{1}$ peak at the potential minimum splits into two peaks (black arrows). b, Constant-current scanning tunneling microscopy topograph of the cleaved surface. Inset shows the atomic-resolution image obtained by scanning the small area. $\mathbf{c}$, Potential landscape of the

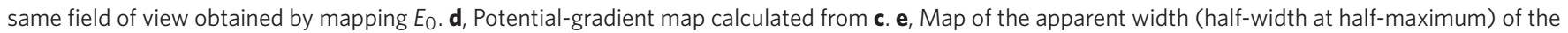
$L_{L}$ peak. These four images (b-e) were taken simultaneously with $V_{s}=+50 \mathrm{mV}, I_{t}=50 \mathrm{pA}$ and $V_{\text {mod }}=2.8 \mathrm{mV}$ rms. (For the inset of $\mathbf{b}, V_{s}=-100 \mathrm{mV}$, $I_{\mathrm{t}}=50 \mathrm{pA}$.) The $\mathrm{LL}_{0}$ peak in the individual spectrum was fitted with a single Lorentzian function to obtain $E_{0}$ and the apparent width of the peak.

a

d
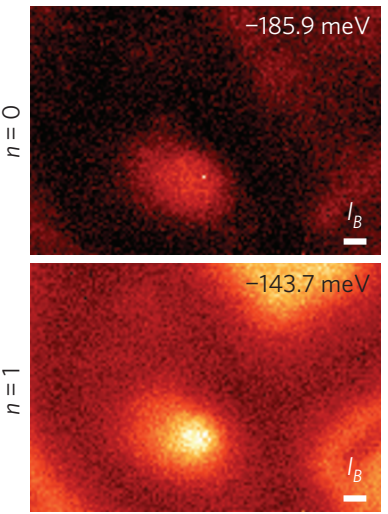

g

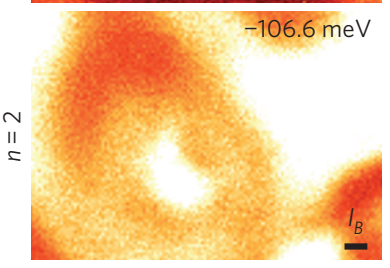

b
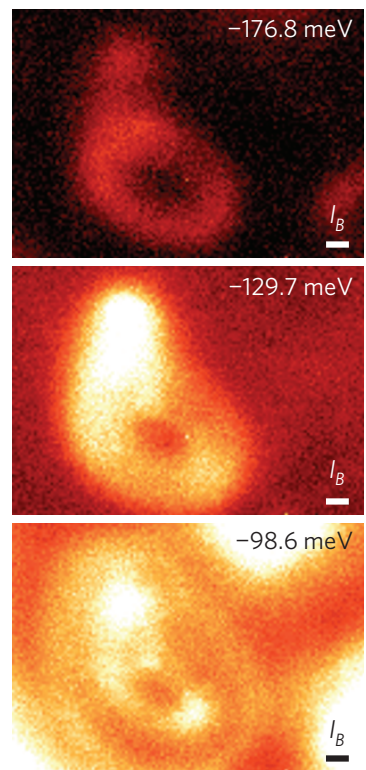

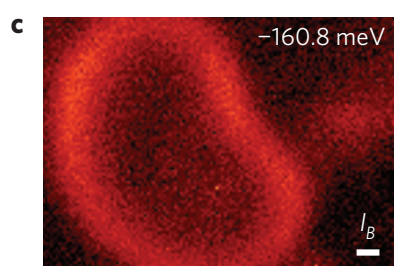

$0.8 \mathrm{nS}$

f

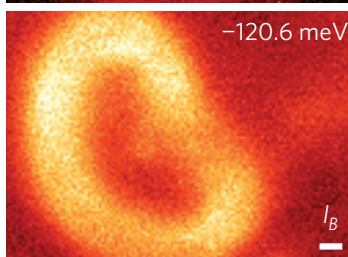

i

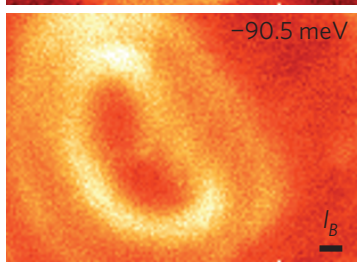

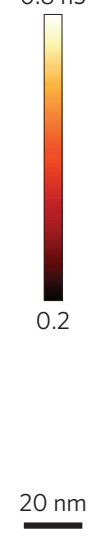

Figure 2 | Spatial and energy evolutions of localized Landau orbits trapped inside the potential dip at $B=11 \mathrm{~T}$. Conductance images taken at different energies exhibit the ring-like trajectory of Landau orbits drifting along the equipotential lines. a-c, $\mathbf{d}-\mathbf{f}$ and $\mathbf{g}$-i are for $L L_{0}, L L_{1}$ and $L L_{2}$, respectively. The complete data set is presented as a movie in the Supplementary Information. The width of the ring increases and the concentric-ring structure becomes evident for $L L_{2}$. The magnetic length $I_{B}$, which is a measure of the size of the $L L_{0}$ orbit, is shown in each panel for comparison.

correspond to different quantum states as described later. Recently, similar splitting has also been observed in graphene $e^{23}$. Interestingly, the $L_{1}$ peak splits into two peaks even at the potential minimum. We will show below that this splitting of the $\mathrm{LL}_{1}$ peak is a direct consequence of the two-component nature.

Next we show the results of SI-STM around the potential minimum. Figure 2 shows a series of conductance maps at $11 \mathrm{~T}$ in the same field of view as in Fig. 1b-e. All the maps exhibit prominent ring-like structures, which are ascribed to the Landau orbits drifting along the equipotential lines ${ }^{17}$. The ring corresponding to the $\mathrm{LL}_{0}$ state emerges at the potential minimum and expands with increasing energy (Fig. 2a-c). With further increasing energy, the ring expands out of the field of view and another ring associated with the $\mathrm{LL}_{1}$ state evolves (Fig. $2 \mathrm{~d}-\mathrm{f}$ ). Expansion of the ring is also observed in the $\mathrm{LL}_{2}$ state (Fig. $2 \mathrm{~g}-\mathrm{i}$ ) and even higher $\mathrm{LL}_{n}$ states (not shown). The ring gets wider with increasing $n$ and splits into two concentric rings for $\mathrm{LL}_{2}$, characterizing the internal structure of Landau orbits. 
a

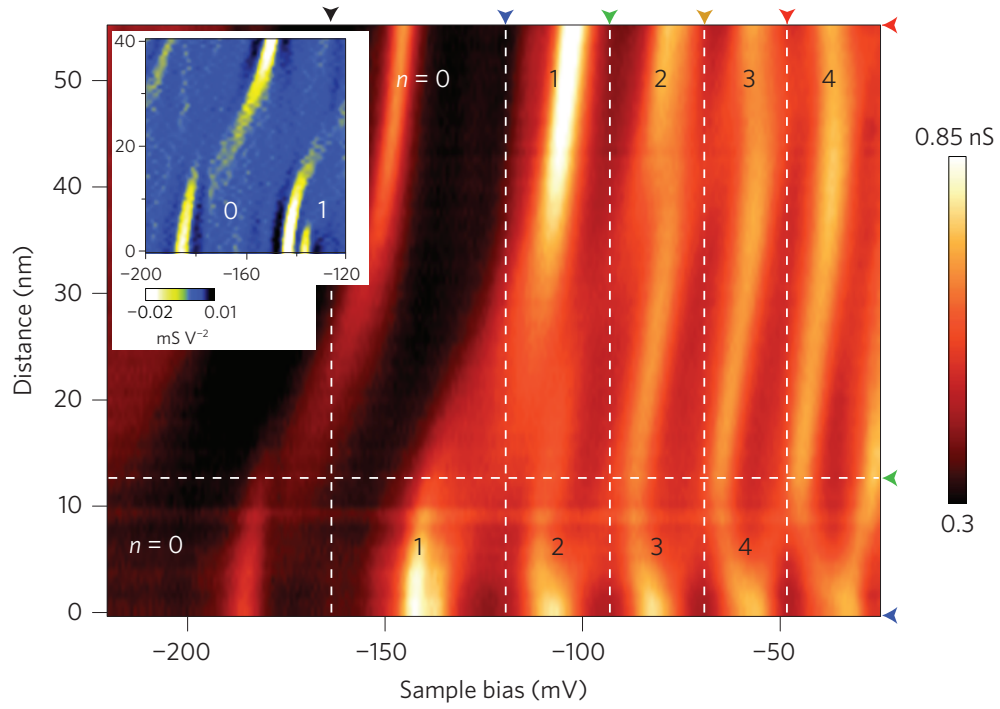

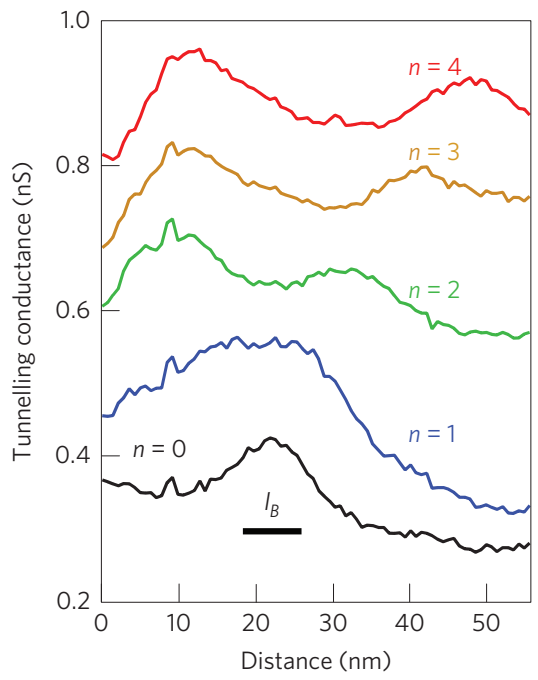

Figure 3 | Branching of Landau subbands and internal structures of Landau orbits at $11 \mathrm{~T}$. a, A false colour plot of the conductance-spectrum evolution from the potential minimum along the line shown in Fig. 1b-e. Inset depicts the second derivative of conductance with respect to the bias voltage, which highlights the splitting features in the lower $(n=0$ and $n=1)$ LLs. Higher LLs evolve smoothly, but split into two apparent branches. Spectra shown in Fig. 1a correspond to the horizontal line-cuts from this panel at distances marked by the horizontal arrows. $\mathbf{b}$, Vertical line-cuts from a at energies marked by the vertical arrows, showing internal structures of drifting Landau orbits for different $n$. Each curve is offset vertically for clarity. Although the number of peaks increases with $n$ in a conventional two-dimensional electron system ${ }^{7}$, there appear at most two peaks in the topological surface state of $\mathrm{Bi}_{2} \mathrm{Se}_{3}$. The scale bar denotes $I_{B}$.

We further investigate the internal structure by analysing a series of conductance spectra taken along the line shown in Fig. 1b-e. As shown in Fig. 3a, the LDOS evolution shows the spatially dispersing Landau subbands. Corresponding to the peak splitting shown in Fig. 1a, $n=0$ and $n=1$ Landau subbands are broken at the potentialgradient maximum and at the potential minimum, respectively. The spatial evolutions of higher $(n>1)$ Landau subbands are smooth but each subband broadens and splits into two apparent branches at the intermediate region, which correspond to the two concentric rings in Fig. 2.

We examined the detailed LDOS distribution across the drifting Landau orbits by taking vertical line-cuts from Fig. 3a (Fig. 3b). As is already seen in Fig. 2, the Landau orbit gets wider with increasing $n$. This behaviour is common to both conventional ${ }^{7}$ and Dirac ${ }^{24}$ systems, because the quantum Larmor radii for $n>0$ LLs, which characterize the widths of the Landau orbits, are given by $l_{B} \sqrt{2 n+1}$ and $l_{B} \sqrt{2|n|}$ for conventional and Dirac systems, respectively (Supplementary Section 1.2).

A remarkable difference between the two systems appears in the internal structures. In the case of conventional systems, the LDOS variation across the drifting $\mathrm{LL}_{n}$ orbit exhibits $n+1$ peaks because the corresponding wavefunction contains $n$ nodes $^{7,25}$ (Supplementary Fig. 2). In contrast, in the case of the topological surface state of $\mathrm{Bi}_{2} \mathrm{Se}_{3}$, the number of peaks never exceeds two, even for $n>1$ LL states, as shown in Fig. 3b.

In the following, we show that our observations can be captured by model calculations and are direct consequences of the two-component wavefunction. We adopt a model Hamiltonian $H=H_{0}+V(\mathbf{r}) \sigma_{0}$, where $H_{0}$ represents the unperturbed Hamiltonian for two-dimensional Dirac electrons in $B$ and $V(\mathbf{r})$ is a circularsymmetric Coulomb potential generated by a subsurface charge $\mathrm{e}^{23} \cdot \sigma_{0}$ is the unit matrix.

It should be noted that the good quantum number here is the total angular momentum $j_{z}$, which is a consequence of strong spin-orbit coupling. This is in contrast to the case of conventional systems, where the orbital angular momentum $l_{z}$ specifies the quantum states $^{25}$. Therefore, $H$ is block diagonalized with respect to $j_{z}$ and we can calculate the energy spectrum $E_{n, j_{z}}$, the wavefunction $\boldsymbol{\Psi}_{n, j_{z}}(\mathbf{r})$, and the LDOS $D(E, \mathbf{r})=\sum_{n, j_{z}} \Gamma /\left(\left(E-E_{n, j_{z}}\right)^{2}+\Gamma^{2}\right)\left|\boldsymbol{\Psi}_{n, j_{z}}(\mathbf{r})\right|^{2}$, assuming a Lorentzian broadening with a damping parameter $\Gamma$. Details are given in the Supplementary Information.

Figure 4a shows an intensity plot of the calculated LDOS as a function of energy and $|\mathbf{r}|$, which reproduces the overall features of the experimental results shown in Fig. 3a. The discrete vertical ridges seen in the $n=0$ Landau subband correspond to the different $j_{z}$ states, which are degenerate for $V(\mathbf{r})=0$. Once $V(\mathbf{r})$ is turned on, this degeneracy is lifted because the Landau orbit with higher $j_{z}$ drifts at larger $|\mathbf{r}|$, where the potential energy is higher.

Figure $4 \mathrm{~b}$ depicts the calculated LDOS spectra at representative points, resembling the observed tunnelling spectra shown in Fig. 1a. In particular, the splitting of the $\mathrm{LL}_{1}$ peak at the bottom of the potential is well captured. The physical picture of this splitting can be understood by looking into the nature of the wavefunction at $\mathbf{r}=0$. By inspecting the functional form of $\boldsymbol{\Psi}_{n, j_{z}}(\mathbf{r})$ given in the Supplementary Information, one finds that $\boldsymbol{\Psi}_{n \neq 0, j_{z}}(\mathbf{r}=0)$ consists of only two quantum states, with $j_{z}=+1 / 2$ and $-1 / 2$, which originate from the up-spin and down-spin components, respectively. Because these two states have different spatial extent, their energies are different; the LDOS peak splits accordingly. Thus, the splitting of the $L_{1}$ peak at $\mathbf{r}=0$ is a direct consequence of the twocomponent nature. The splitting should also occur for $\mathrm{LL}_{n}$ with $n>1$, but its detection is much harder because the energy difference between $j_{z}= \pm 1 / 2$ states becomes smaller with increasing $n$. Note that the $\mathrm{LL}_{0}$ peak does not split at $\mathbf{r}=0$ because only the downspin component of $\boldsymbol{\Psi}_{0, j_{z}}(\mathbf{r}=0)$ is non-zero. The relevance of this scenario is highlighted by looking at the spin-resolved LDOS at $\mathbf{r}=0$ (Fig. 4b).

The two-component nature also explains the absence of nodal structure in the LDOS distributions. The $|\mathbf{r}|$-dependence of the calculated LDOS (Fig. 4c, thick black curves) exhibits only two peaks for $n>0$, being in agreement with the experiment. We also plot the spin-resolved partial LDOS associated with the eigenstate at a given energy. Such a 'dominating eigenstate' mainly contributes to the total LDOS, albeit other states also participate if $\Gamma$ is finite 
a

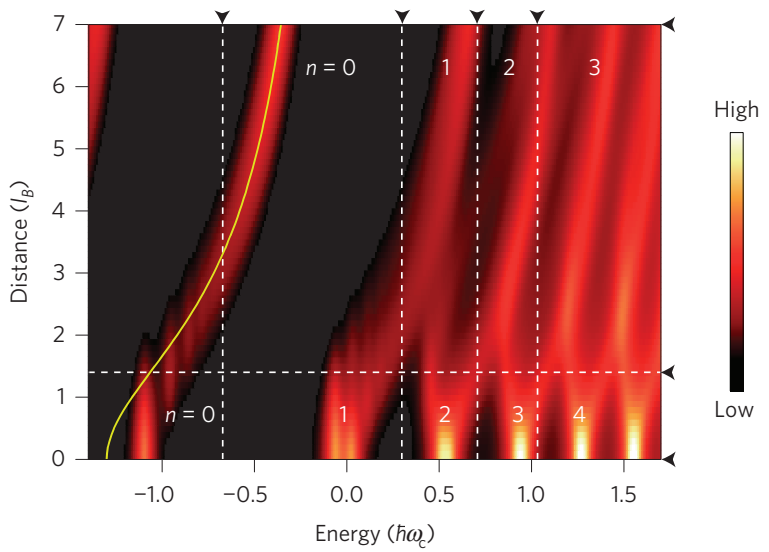

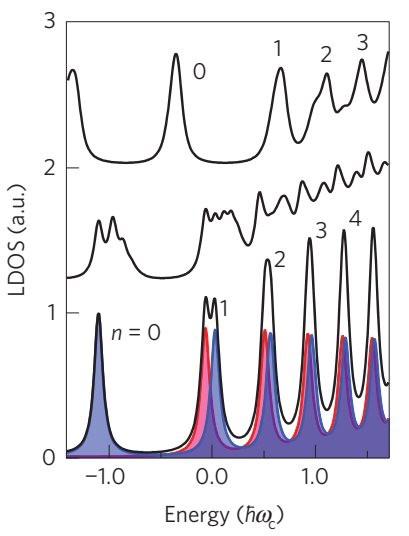

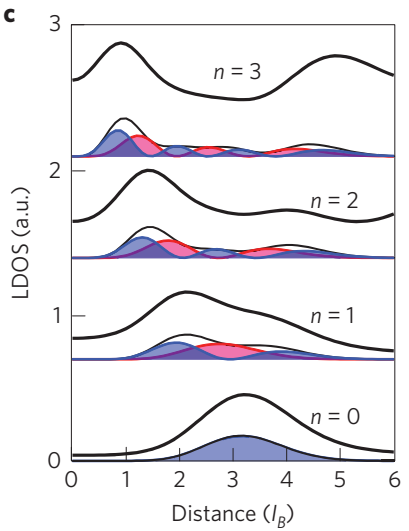

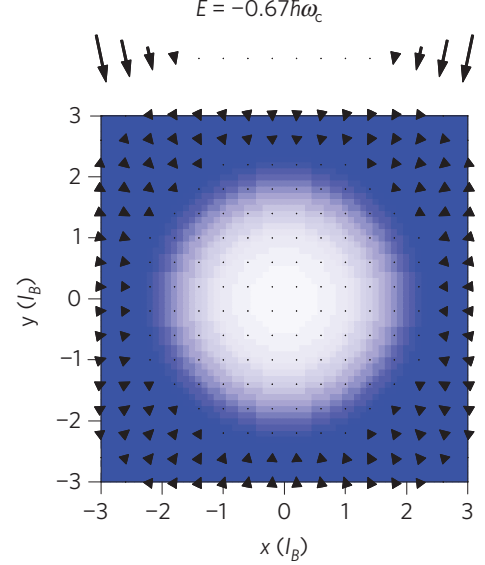

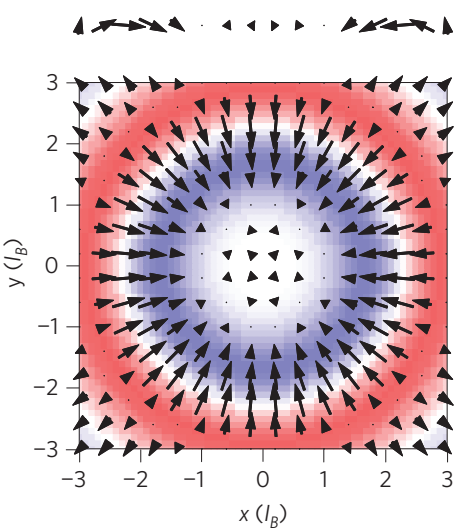

$\mathbf{f}$

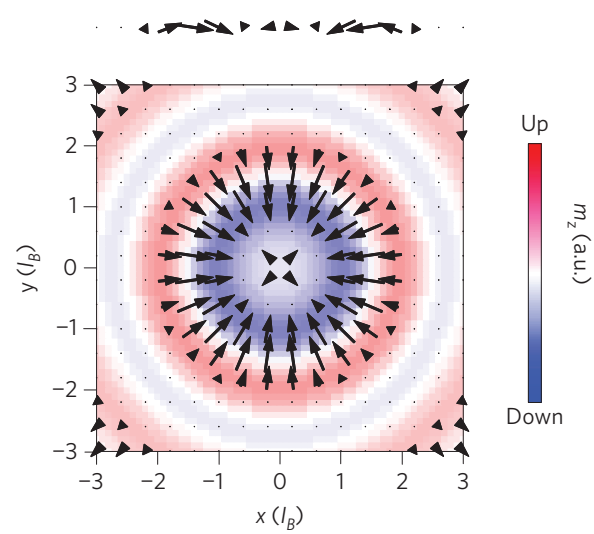

Figure 4 | Results of model calculations based on the two-component Dirac Hamiltonian. a, Intensity plot of the calculated LDOS as a function of energy and distance from the bottom of the potential. The yellow solid line denotes the radial variation of the potential used for the calculation. The length is measured in units of $I_{B}$ and the energy is measured in units of $\hbar \omega_{C}$, where $\omega_{C}=\sqrt{2} v / I_{B}$ is the cyclotron frequency and $v$ is the electron velocity. The damping parameter $\Gamma$ was set to $0.05 \hbar \omega_{c}$. See Supplementary Section 1.2 for details. b, LDOS spectra, in arbitrary units (a.u.), obtained by taking horizontal line-cuts at the representative points shown by horizontal arrows in $\mathbf{a}$. (From bottom to top, distance $|\mathbf{r}|=0,1.4 I_{B}$ and $7.0 I_{B}$, respectively.) Each curve is offset vertically for clarity. At the bottom of the potential, partial LDOS spectra associated with the up-spin (filled red curve) and down-spin (filled blue curve) components are also shown. It is clear that $L L_{0}$ consists of a down-spin component only and the splitting of the $L L_{1}$ peak is associated with the spin degrees of freedom. $\mathbf{c}$, Thick solid lines represent internal structures of Landau orbits obtained by taking vertical line-cuts at the representative energies shown by vertical arrows in a. Partial LDOS (thin black lines) from the dominating eigenstate and its up-spin (filled red curves) and down-spin (filled blue curves) components are also shown. Data for each $n$ are offset vertically for clarity. Nodes in the up-spin component are filled by the down-spin component and vice versa. $\mathbf{d}-\mathbf{f}$, Spatial distribution of energy-dependent spin-magnetization vectors defined by $m_{i}=\hbar / 2 \sum_{n, j_{z}} \Gamma /\left(\left(E-E_{n, j_{2}}\right)^{2}+\Gamma^{2}\right) \Psi_{n, j_{z}}^{\dagger}(\mathbf{r}) \sigma_{i} \boldsymbol{\Psi}_{n, j_{2}}(\mathbf{r})$, where $\sigma_{i}(i=x, y, z)$ are Pauli matrices. The in-plane components are indicated by arrows and the out-of-plane component is indicated by colours. The line-cut at $y=0$ is also shown above each panel.

(Supplementary Section 1.5). Although the down-spin component (blue) has $n$ nodes, as in the case of conventional systems, the number of nodes for the up-spin component (red) is $n-1$. Therefore, the nodes for one component are always filled by the other, and two enhanced LDOS peaks are formed near the edges. These features are also expected in other two-dimensional Dirac systems such as graphene.

A unique feature of the topological surface states is that the potential variation not only affects the orbital motion but also induces non-trivial spin-magnetization textures through the strong spinorbit coupling. Indeed, calculated spin-magnetization distributions shown in Fig. 4d-f exhibit energy-dependent cycloidal-helix-like patterns along the radial direction. A future spin-polarized STM experiment may detect these patterns directly. Such an emergence of spin-magnetization textures is not expected in graphene, where the Dirac electron nature is associated with the sublattice degrees of freedom. We anticipate that the combination of Landau quantization and a tailored potential landscape in the topological surface states may provide a novel 'magnetoelectric' control of spins, leading to intriguing spintronic and topological applications.

\section{Methods}

$\mathrm{Bi}_{2} \mathrm{Se}_{3}$ crystals were prepared by the melt-growth technique. SI-STM experiments were performed at $1.5 \mathrm{~K}$ with a commercial low-temperature ultrahigh-vacuum STM (Unisoku USM-1300) modified by ourselves ${ }^{26}$. The clean and flat surface was obtained by in situ cleaving at $\sim 77 \mathrm{~K}$. After the cleaving, the sample was transferred quickly to the STM unit, which was kept below $10 \mathrm{~K}$. Magnetic field was applied perpendicular to the cleaved surface. An electro-chemically etched tungsten wire was used as an STM tip, which was cleaned and characterized in situ with a field-ion microscope. Tunnelling spectra were taken with a software lock-in detector integrated in a commercial STM controller (Nanonis).

Received 10 February 2014; accepted 4 August 2014; published online 14 September 2014

\section{References}

1. Geim, A. K. \& Novoselov, K. S. The rise of graphene. Nature Mater. 6, 183-191 (2007). 
2. Castro Neto, A. H. et al. The electronic properties of graphene. Rev. Mod. Phys. 81, 109-162 (2009).

3. Tajima, N. et al. Transport properties of massless Dirac fermions in an organic conductor $\alpha$-(BEDT-TTF) ${ }_{2} \mathrm{I}_{3}$ under pressure. Europhys. Lett. 80, 47002 (2007).

4. Ran, Y. et al. Nodal spin density wave and band topology of the FeAs-based materials. Phys. Rev. B 79, 014505 (2009).

5. Hasan, M. Z. \& Kane, C. L. Topological insulators. Rev. Mod. Phys. 82, 3045-3067 (2010).

6. Qi, X-L. \& Zhang, S-C. Topological insulators and superconductors. Rev. Mod. Phys. 83, 1057-1110 (2010)

7. Hashimoto, K. et al. Robust nodal structure of Landau level wave functions revealed by Fourier transform scanning tunneling spectroscopy. Phys. Rev. Lett. 109, 116805 (2012)

8. McClure, J. W. Diamagnetism of graphite. Phys. Rev. 104, 666-671 (1956).

9. Goerbig, M. O. Electronic properties of graphene in a strong magnetic field. Rev. Mod. Phys. 83, 1193-1243 (2011).

10. Novoselov, K. S. et al. Two-dimensional gas of massless Dirac fermions in graphene. Nature 438, 197-200 (2005).

11. Zhang, Y., Tan, Y-W., Stormer, H. L. \& Kim, P. Experimental observation of the quantum Hall effect and Berry's phase in graphene. Nature 438, 201-204 (2005)

12. Li, G., Luican, A. \& Andrei, E. Y. Scanning tunneling spectroscopy of graphene on graphite. Phys. Rev. Lett. 102, 176804 (2009).

13. Miller, D. L. et al. Observing the quantization of zero mass carriers in graphene. Science 324, 924-927 (2009).

14. Hanaguri, T. et al. Momentum-resolved Landau-level spectroscopy of Dirac surface state in $\mathrm{Bi}_{2} \mathrm{Se}_{3}$. Phys. Rev. B 82, 081305(R) (2010).

15. Cheng, P. et al. Landau quantization of topological surface states in $\mathrm{Bi}_{2} \mathrm{Se}_{3}$. Phys. Rev. Lett. 105, 076801 (2010).

16. Morgenstern, M., Klijn, J., Meyer, Chr. \& Wiesendanger, R. Real-space observation of drift states in a two-dimensional electron system at high magnetic fields. Phys. Rev. Lett. 90, 056804 (2003).

17. Hashimoto, K. et al. Quantum Hall transition in real space: From localized to extended states. Phys. Rev. Lett. 101, 256802 (2008).

18. Miller, D. L. et al. Real-space mapping of magnetically quantized graphene states. Nature Phys. 6, 811-817 (2010).

19. Niimi, Y. et al. Real-space imaging of alternate localization and extension of quasi-two-dimensional electronic states at graphite surfaces in magnetic fields. Phys. Rev. Lett. 97, 236804 (2006).
20. Chen, Y. L. et al. Massive Dirac fermion on the surface of a magnetically doped topological insulator. Science 329, 659-662 (2010).

21. Zhang, Y. et al. Origin of spatial charge inhomogeneity in graphene. Nature Phys. 5, 722-726 (2009).

22. Beidenkopf, H. et al. Spatial fluctuations of helical Dirac fermions on the surface of topological insulators. Nature Phys. 7, 939-943 (2011).

23. Luican-Mayer, A. et al. Screening charged impurities and lifting the orbital degeneracy in graphene by populating Landau levels. Phys. Rev. Lett. 112, 036804 (2014).

24. Okada, Y. et al. Visualizing Landau levels of Dirac electrons in a one-dimensional potential. Phys. Rev. Lett. 109, 166407 (2012).

25. Yoshioka, D. Local density of states around impurity in a strong magnetic field: I. Two-dimensional system with parabolic dispersion. J. Phys. Soc. Jpn 76, 024718 (2007)

26. Hanaguri, T. Development of high-field STM and its application to the study on magnetically-tuned criticality in $\mathrm{Sr}_{3} \mathrm{Ru}_{2} \mathrm{O}_{7}$. J. Phys.: Conf. Ser. 51, 514-521 (2006)

\section{Acknowledgements}

The authors thank X. Chen, Y. L. Chen, K. Iwaya, Y. Kohsaka, K. Nomura, M. Ogata, Y. Okada and A. W. Rost for discussions. This work was partly supported by Grant-in-Aid for Scientific Research from the Ministry of Education, Culture, Sports, Science and Technology of Japan (Grant No. 23103519).

\section{Author contributions}

Y-S.F. carried out the experiments and M.K. performed the theoretical modelling and calculations. $\mathrm{Bi}_{2} \mathrm{Se}_{3}$ single crystals were grown by K.I. and T.S. T.H. and H.T. supervised the project. Y-S.F., M.K. and T.H. designed the experiment, analysed the data and wrote the manuscript.

\section{Additional information}

Supplementary information is available in the online version of the paper. Reprints and permissions information is available online at www.nature.com/reprints.

Correspondence and requests for materials should be addressed to T.H.

\section{Competing financial interests}

The authors declare no competing financial interests. 